RECENT ADVANCES IN CROHN'S DISEASE 


\section{DEVELOPMENTS IN GASTROENTEROLOGY}

VOLUME 1

Also in this series:

2. Motta PM, Didio LJA, eds: Basic and clinical hepatology. 1981. ISBN 90-247-2475-9

series ISBN 90-247-2441-4 


\section{RECENT ADVANCES IN CROHN'S DISEASE}

Proceedings of the 2nd International Workshop on Crohn's Disease, Noordwijk / Leiden, 25-28 June 1980

edited by

A.S. PEÑA

Department of Gastroenterology,

State University of Leiden, Leiden,

The Netherlands

\section{IRENE T. WETERMAN}

Department of Gastroenterology,

State University of Leiden, Leiden,

The Netherlands

C.C. BOOTH

Clinical Research Centre,

Northwick Park, Harrow,

U.K.

\section{W. STROBER}

National Cancer Institute,

National Institutes of Health, Bethesda, MD,

U.S.A.

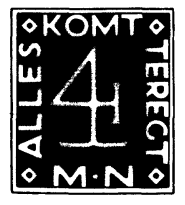

1981

MARTINUS NIJHOFF PUBLISHERS

THE HAGUE / BOSTON / LONDON 
Distributors :

for the United States and Canada

Kluwer Boston, Inc.

190 Old Derby Street

Hingham, MA 02043

USA

for all other countries

Kluwer Academic Publishers Group

Distribution Center

P.O. Box 322

3300 AH Dordrecht

The Netherlands

This volume is listed in the Library of Congress Cataloging in Publication Data

ISBN-13: 978-94-009-8275-8

e-ISBN-13: 978-94-009-8273-4

DOI: $10.1007 / 978-94-009-8273-4$

Published February 1981.

Copyright (c) 1981 by Martinus Nijhoff Publishers bv, The Hague.

Softcover reprint of the hardcover Ist edition 1981

All rights reserved. No part of this publication may be reproduced, stored in a retrieval system, or transmitted in any form or by any means, mechanical, photocopying, recording, or otherwise, without the prior written permission of the publisher

Martinus Nijhoff Publishers bv, P.O. Box 566, 2501 CN The Hague, The Netherlands. 


\section{FOREWORD}

The study of disease entities as complex as Crohn's disease will increasingly require comprehensive knowledge of formerly unrelated areas of the medical sciences. To promote this broad approach, a conference was organized in which geneticists, morphologists, immunologists, and virologists participated as well as clinicians whose work is focused on Crohn's disease. Ample time was given to the presentation of major new findings in each of these areas, and comments were given by the participants in the various sections. This approach yielded many new ideas, because individuals with very different backgrounds were able to address old problems from fresh angles.

This volume, published in January, 1981, contains the papers presented during the workshop held in June, 1980, in Noordwijk/Leiden, The Netherlands. In addition, we have included the extensive discussions, edited by experts in the field, which followed each presentation. Finally, each main category is followed by a summary of the topic covered as well as many valuable conclusions concerning the significance of recent work and ideas for new directions in research. In the Preface, J.B. Kirsner gives a comprehensive review of the material, and in an Afterword A.J.Ch. Haex deals with a number of other aspects.

We believe that this volume will be of value to a wide spectrum of scientists and clinicians and that it will help to establish the multidisciplinary approach to Crohn's disease as the one that should predominate in the future. 


\section{CONTENTS}

Preface

Section A

Section B

Section C

Section D

Section E

Section F

Section G

Section $\mathrm{H}$

Section Editor: R.B. McConnell

Closing Remarks A.J.Ch. Haex

Author Index 


\title{
PREFACE
}

by Joseph B. Kirsner

The Louis Block Distinguished Service Professor of Medicine University of Chicago, Chicago, U.S.A.

The "Coming of Age" of Crohn's disease

\begin{abstract}
"Natura non facit saltus"
Nature does not proceed by leaps, but rather reveals its secrets slowly, quitely and grudgingly. Notable advances of today have their background in work often carried out decades before.

Carl Linnaeus, Philosophica Botanica, (Section 77), 1751
\end{abstract}

It is perhaps difficult in 1980 to realize that not too long ago, Crohn's disease was an unknown entity; and that for many years after its classic description by Crohn, Ginsberg and Oppenheimer in 1932, this entity was infrequently discussed and rarely investigated. Today, Crohn's disease is regarded as one of the more challenging problems in medicine; in part, because of its rising incidence in many parts of the world; in part, because of the intriguing associated systemic problems and in part, because Crohn's disease clinically and investigationally involves so many scientific disciplines: internist, psychiatrist, pharmacologist, gastroenterologist, radiologist, surgeon, pathologist, immunologist, geneticist, biochemist, cell biologist, among other basic sciences. 
The immediately impressive feature of this book then is that it is a compilation of nearly 100 papers presented in a three-day meeting in The Netherlands in June 1980, attended by approximately 100 physicians and scientists; a constellation of topics and participants that would not have been possible a few years earlier. This workshop also is a testimony to the highly informed administrative talent of the programs' organizers: Prof.Dr. A.J.Ch. Haex and Dr. A.S. Peña of Leiden, The Netherlands, who selected the speakers and arranged the meeting. In refreshing contrast to the usual international symposium, the emphasis here is upon recent or newer observations, especially in the areas of epidemiology, histopathology, genetics, microbiology, immunology, and some aspects of therapy. As such, the book contains much valuable information on the nature of Crohn's disease; especially of interest to the investigator. The organization of the material, as shown in the contents, reflects currently attractive research areas.

Though focussed on selected research aspects of Crohn's disease, the individual presentations and the subsequent discussions reflect an awareness; perhaps an anticipation of additional knowledge yet to come. Where then are we today in our understanding of Crohn's disease?

We know more of its clinical features and its course than ever before. An enormous number of complications; local and systemic; now have been identified; in themselves intriguing pathogenetically since they implicate the diseased bowel and its internal milieu as the source of these difficulties. They also direct attention to concepts of individual vulnerability and individual resistance to both the bowel disease and the associated systemic disorders. However, the underlying cause or causes of Crohn's disease remain obscure; and this continuing deficiency provides the motivation for these proceedings.

Although several recent papers report a declining incidence (Aberdeen, Scotland and Stockholm county, Sweden), the rising incidence of Crohn's disease elsewhere in the world is 
intriguing; all the more so in view of the similar clinical features of Crohn's disease everywhere, despite different ethnic populations, environmental circumstances, dietary habits and socio-cultural customs. Possible explanations have included some type of virus, unusual microbial agents, dietary constituent, (i.e., excessive intakes of sugar), environmental pollutant, tobacco or contraceptive steroids; but without decisive supporting evidence. Carefully organized epidemiological studies of Crohn's disease, except in a few discrete geographic areas have been lacking; studies that could provide useful clues for further investigations. Epidemiological studies and other cooperative clinical projects would be greatly enhanced by more easily applied and more uniform criteria of diagnosis, and by generally acceptable quantified assessment of clinical activity. Despite considerable application in the United States via the National Cooperative Crohn's Disease Study [NCCDS], the Crohn's disease activity index has not proved to be an easily utilized measure of disease activity. The initial part of this workshop, directed to this particular question therefore considers an important practical problem. The challenge is especially great because of the absence of any single pathognomonic feature or laboratory test of Crohn's disease, the relatively non-specific clinical and histopathological manifestations, and because of the associated clinical problems. Despite their many similarities, the two entities: Crohn's disease and ulcerative colitis, correctly should be separated in any consideration of inflammatory bowel disease; for this object to be attained. Despite current limitations, the more uniform objective assessment of clinical activity should be an attainable goal, worthy of further effort. Once achieved, the information should accelerate future cooperative clinical studies of Crohn's disease.

More complete morphologic understanding of the Crohn's tissue reaction always has been accepted as a potentially clarifying approach to the nature of the disease. Knowledge in this area has been limited by the available methodology; 
a deficiency now being remedied in part by more careful light microscopy and by the use of transmission and scanning electron microscopy, histochemical and immunohistochemical studies. These approved techniques reveal the intensity of the crohn's inflammatory reaction, affecting every constituent of the bowel wall [epithelial cells, blood vessels, muscle layers and neural elements), multiple cellular types, including polymorphonuclear leucocytes, lymphocytes, eosinophils, basophils, mast cells, Paneth cells and macrophages; and the widely disseminated though focal distribution of the identifiable Crohn's lesion. A handicap of the present morpholocigal approach is imposed by current limitations in knowledge, as to what to look for, how to seek it and where to look. The granuloma, though easily recognized and often emphasized as diagnostic, is not specific to Crohn's disease. Granulomas are not evident in 50 percent of patients; their regional distribution varies and, apparently, once having been present, they may disappear. While some animal transmission studies have utilized the granuloma and the site of the "causative agent" of Crohn's disease, decisive supportive evidence has not appeared. Whether or not the granuloma reflects an adaptive protective response of the host to an etiological agent awaits much more knowledge of both Crohn's disease and host defenses in general.

The specificity of the histologic changes also remains in question. The focal nature of the early Crohn's lesion, its scattered distribution along the entire gastrointestinal tract, and the micro-ulcerations of the dome epithelium of the Peyer's patches on the other hand, are observations of potential etiologic significance; perhaps compatible with an unusual viral or microbial agent but also perhaps corresponding to the "focal" distribution of the lymphoid follicles in the bowel wall. Future studies of the nature of the Crohn's disease tissue reaction should evaluate not only the possible role of mast cells and Paneth cells, but also there the prostanoids, thromboxane, lysosomal enzymes of monocytic origin; and intestinal endorphins and enkephalins. 
The search for pathogenic bacteria, viruses and other microbial agents has been in progress since the entity was first described. Beginning with the exclusion of the tubercle bacillus, then Shigella, E. histolytica and lymphopathia venereum virus, all hitherto known bacterial, viral and parasitic agents appeared to have been eliminated. However, the recent identification of "new" bacterial causes of enteritis and colitis i.e., Yersinia Pseudotuberculosis, Campylobacter fetus ss. jejuni, and Aeromonas hydrophila has rekindled microbiological interest in Crohn's disease. Today, the microbiological focus is upon such anaerobic organisms, as Eubacteria and Peptostreptococcus [strains $\mathrm{C}_{18^{\prime}} \mathrm{Me}_{46} \mathrm{M}^{\prime} \mathrm{Me}_{47}$ ] cell-wall defective, Pseudomonas-like bacteria, various strains of E. coli, Mycobacteria and Mycoplasma. However, convincing data for the etiological involvement of any infectious agent in Crohn's disease has not been published.

Microbial interest has centered also upon a possible viral etiology; based upon such indirect information as the presence of lymphocytotoxic antibodies in the family household contacts of Crohn's patients; and the apparent presence of a "transmissible agent" the cytopathic constituent in Crohn's disease tissue injected into animals [mouse, rabbit]. However, while some observations are intriguing, including either the development of ulcerative colitis or Crohn's disease among married couples [five such reports], the evidence to date is inconclusive. The demonstration of similar in vitro cytopathic effects, though less often, with non-IBD intestinal tissues, the negative results of other investigators utilizing a variety of virological techniques, the inability to reproduce the disease with cultured agents obtained from resected Crohn's tissue, the inability to identify viral particles or intraepithelial microorganisms on electron microscopy and the non-fulfillment of Koch's postulates, all of these observations indicate the need for restraint in the acceptance of the viral etiology. Nevertheless, the demonstration of replicating, transmissible cytopathic agents in Crohn's disease tissue is at least worthy of further cooper- 
ative examination; especially with more subtle virus-demonstrating techniques. All that is known of Crohn's disease clinically and histopathologically, is compatible with microbiological involvement of some type. Microbiological concepts worthy of further study might include an "imbalance" of the normal entero-colonic microflora, and hypersensitivity of the gut-associated immune apparatus to constituents of the endogenous normal bacterial flora, and to bacterial products [lipopolysscharides, endotoxins].

The possible involvement of the immunological mechanism in inflammatory bowel disease, first proposed by Kirsner and Palmer in 1954, continues to be a highly active research area. The immunological studies to date have demonstrated a central role of the gastrointestinal tract in the immunological homeostasis of the body and the importance of the gutassociated lymphoid tissue and the locally protective role of secretory IgA at the gastrointestinal epithelial surface. Although immunological processes probably play some role in Crohn's disease, an immunological basis for this disease has not been established. The many immunological observations [both humoral and cellular] in Crohn's disease at present are confusing and difficult to interpret; including circulating antibodies against bacterial, viral, food, human and rat colon, as well as enterobacterial and various tissue antigens, defective cutaneous anergy, the variations in $B$ and $T$ cell numbers and proportions, imbalances in various $T$ cell subsets [normal total $T$ cell numbers and marked decreases in proportion and absolute numbers of the cells bearing Fc receptors for IgM], IgM-type lymphocytotoxic antibodies against B cells, the absence of a consistent correlation between major immunoglobulin classes and the activity of the inflammatory bowel disease, the variable lymphocyte responses to mitogens, the possible presence and the role of circulating immune complexes, the increased monocytic lysosomal enzyme activity, the alterations in complement [elevated $\mathrm{C}_{3}, \mathrm{C}_{4}$ and increased metabolism of $\mathrm{Cl}_{\mathrm{q}}$ and $\mathrm{C}_{3}$ and the activation of the alternate complement pathway]; all of these findings, and 
others, appear to be secondary epiphenomena rather than primary etiologically significant features of the disease. Since studies of the blood do not necessarily reflect the local immunological status of the gastrointestinal tract, techniques are being developed for isolating and assessing the comparable immunobiological activities of non-epithelial cell populations obtained from the intestinal wall; especially lymphocytes and macrophages. Such studies have demonstrated increased numbers of $B$ type lymphoid cells in the intestinal infiltrates, $T$ cells, and variable numbers of IgA, IgM, IgG, IgD and IgE cells in the lamina propria; "killer" lymphocytes, and other lymphocyte subsets. Since the present methodology does not permit the separation of "pure" lymphocytes, the studies to date on mixed lymphocytes populations, probably have limited significance. Nevertheless, further investigation of gut mucosal immune defenses, including antigenic penetration of the bowel mucosa, and possible defect in the mucosal secretory [IgA] immune system are attractive approaches. The presence of elements of the complement system, $\left[\mathrm{Cl}_{\mathrm{q}}, \mathrm{C}_{3}\right]$ and increased number of immunoglobulin-containing cells and immune complexes in Crohn's disease tissue are compatible with a local immune mediated reaction, perhaps in genetically-determined vulnerable individuals.

The concept of an individual [genetic] predisposition to IBD derives from the selective occurrences of IBD after enteric [food poisoning] and viral infections [infectious mononucleosis], the increased incidence of Ankylosing spondylitis, a disorder with an established autosomal dominant gene mechanism, and especially from the observed multiple instances of IBD in the same family. Numerous surveys now have documented the familial occurrence of Crohn's disease in at least 20 percent and up to 35 percent of patients, exceeding the most generous estimates of IBD incidence in the general population. The familial tendency appears stronger in Crohn's disease than ulcerative colitis. Most of the familial occurrences are in one additional family member, but numerous families of three or more, up to eight affected members have 
$x v i$

been reported. First-degree relatives are more vulnerable. The intermingling of these two disorders among family members is an intriguing feature. At least 11 reports have documented the occurrence of Crohn's disease among monozygotic twins. No genetic markers have been identified in Crohn's disease; and inheritable protein, enzymatic or metabolic defects or chromosomal abnormalities have not been demonstrated. The chromosomal changes reported thus far appear to be non-specific, possibly attributable to the associated under nutrition, yet unidentified metabolic alterations or to the many potent medications prescribed. The genetic mechanism implicated in the individual vulnerability to IBD are not known. The most likely explanation appears to be the combined interaction or multiple genes, the multifactorial or polygenic type of inheritance. The genetic contribution presumably would be to facilitate a state of susceptibility, locally and systemically, with the precipitation of crohn's disease by varied external environmental circumstances. The nature of the susceptibility is not known. No consistent association has been found thus far between specific HLA antigens [HLA-A, $-B,-C$, or -DR locus antigens] and Crohn's disease, except for the positive correlation with HLA-B27 in those patients with ankylosing spondilytis or acute iritis. However, the possibility of other associations, positive or negative, with other HLA antigens awaits further investigations, especially since present studies have dealt with only a small portion of the human genome. This research area is all more attractive in view of the possible relationship between elements of the human genome and immunoregulatory mechanisms.

The treatment of Crohn's disease continues to involve arbitrary approaches. We are more aware today of the limitations of the medical and surgical therapy. In view of the frequent recurrences, operations now are reserved chiefly for serious complications of the disease, and the tendency is toward limited rather than radical bowel resection. In medical management, dependence upon sulphazalasine and steroids alone [usually prednisone] alone often is insufficiently to 
control Crohn's disease continuously. Experience support the usefulness of a program of management, including emotional and physical rest, the restoration and maintenance of optimal nutrition as essential to an adequate response to medications; symptomatic measures to review abdominal discomfort and diarrhea ["steroids-savers"], and the judicious use of antibacterial, anti-inflammatory and "immuno-suppresive" drugs, each adapted to individual responses. For many drugs i.e., sulphazalasine and metronidazole the rationale is unclear; for "immuno-suppresive" medication, requiring up to six months for recognizable therapeutic benefit, whose allegedly immuno-suppresive effects in Crohn's disease have not been demonstrated, and for drugs such as superoxide dismutase and d-penicillamine, whose biological properties and clinical effects are poorly understood, we can only speculate on their therapeutic advantages and limitations. The "scientifically" more acceptable approach of "controlled clinical trials" thus far has failed to provide new insights into the nature of Crohn's disease as revealed by the therapeutic response. The various therapeutical measures currently recommended for Crohn's disease probably have no specific influence upon the tissue reaction per se; but rather, a dampening or inhibiting effect upon the inflammatory/immune crohn's process; applied simultaneously with efforts to improve host defenses. Clinical improvement does seem attributable more to the individual capacity to contain the disease than to any "specific" drug effect. If this concept is valid, then future studies of Crohn's disease should emphasize fundamental investigations of systemic and local [gut] host defenses, the nature of immunocompetence and immunoregulation; the relationship of human immune response genes to the MHC chromosomal region and to the body's defense against microbial and viral agents, and how these mechanisms may influence the individual's response to endogenous and exogenous microbial agents. There already is evidence of an increased susceptibility to certain infections among individuals bearing a given B lymphocyte alloantigen; and japanese investigators have succesfully identified 
HLA-linked control of immune responses to microbial antigens. The combination of microbial factors, host immune responses and genetic influences will encompass the more likely mechanisms in the pathogenesis of IBD.

This approach then is perhaps the major "message" of these proceedings. None of the concepts advanced here probably will endure additional scientific scrutiny. Nevertheless, they document the broadening scientific interest in Crohn's disease, and they examine at least preliminary, some newer ideas. The proceedings also demonstrate the involvement of more, better prepared researches in the study of Crohn's disease than in the past; a major advance. The Second International Workshop on Crohn's Disease thus constitute a "portal of entry" to research in progress, as well as to research in the future. As such, it documents the "coming of age", the blossoming of Crohn's disease as a medical disorder worthy of the most careful clinical and scientific investigation. Such developments alone are sufficient to renew the hope that the 1980 Holland Workshop, by encouraging more perceptive research by well-trained investigators, eventually will increase the basic knowledge of crohn's disease; a necessary prelude to the more effective control and perhaps even the cure of what has emerged as one the more challenging medical disorders of our time. 